Revista de Matemática: Teoría y Aplicaciones 2(2): 59-65 (1995)

\title{
SOBRE EL TEOREMA TAUBERIANO DE W. RUDIN
}

\author{
MARIELOS MORA ${ }^{1}$
}

\begin{abstract}
Resumen
Usando el método de Rudin demostramos que el teorema tauberiano no sólamente es cierto para el núcleo de Poisson, sino para muchos otros núcleos que tengan ciertas características. Así mismo, demostramos un teorema abeliano, que no es otra cosa que el inverso del teorema tauberiano.
\end{abstract}

Abstract

Using W. Rudin's method it is shown that the tauberian theorem can be generalized to several kernels other than the Poisson kernel. We also proof an inverse of the tauberian theorem, that is, an abelian theorem.

\section{Introducción}

Denotemos por $B(0, r) \subset \mathbb{R}^{n}$ la bola abierta centrada en el origen de coordenadas y de radio $r$, y sea $m$ la medida de Lebesgue en $\mathbb{R}^{n}$ normalizada de tal manera que

$$
m(B(0, r))=r^{n} .
$$

En [7], el autor demuestra el siguiente resultado:

\section{Teorema 1.}

Sea $\mu$ una medida de Borel finita y positiva, definida en $\mathbb{R}^{n}$, y sea

$$
u(x, \varepsilon)=\int_{\mathbb{R}^{n}} \frac{c \varepsilon}{\left(|x-\xi|^{2}+\varepsilon^{2}\right)^{\frac{n+1}{2}}} d \mu(\xi), \quad x \in \mathbb{R}^{n}, \varepsilon>0 .
$$

Si existe el límite

$$
\left.\lim _{\varepsilon \rightarrow 0} u(0, \varepsilon)=L \in\right] 0,+\infty[
$$

entonces

$$
\lim _{r \rightarrow 0} \frac{\mu B(0, r)}{m B(0, r)}=L
$$

Es bien sabido que la condición (2) del teorema anterior implica la condición (1), aún en el caso en que $\mu$ es una carga. Por esto el teorema anterior es tauberiano, y el rol de la condición tauberiana lo juega la positividad de la medida $\mu$.

En este trabajo vamos a presentar una generalización del teorema recién citado.

\footnotetext{
${ }^{1}$ Escuela de Matemática, Universidad de Costa Rica
} 


\section{Sección 1.}

Sea $g$ una función continua y acotada definida en el intervalo no acotado $[0,+\infty[$ que satisface

$$
\int_{0}^{+\infty}|g(r)| r^{n-1} d r<+\infty
$$

Denotemos por $\varphi(x)$ la función definida por $\varphi(x)=g(|x|)$, donde $x \in \mathbb{R}^{n}$.

La función $\varphi$ es sumable en todo $\mathbb{R}^{n}$, y vamos a suponer que

$$
\int_{\mathbb{R}^{n}} \varphi(x) d \mu=1
$$

$\varphi$ da origen a una integral singular, que está definida por

$$
\widetilde{\mu_{\varepsilon}}=\frac{1}{\varepsilon^{n}} \int_{\mathbb{R}^{n}} \varphi\left(\frac{x}{\varepsilon}\right) d \mu(x), \quad \varepsilon>0 .
$$

Es fácil ver que en el caso en que

$$
g(r)=\frac{c_{n}}{\left(1+r^{2}\right)^{\frac{n+1}{2}}} ; r=|x|
$$

entonces $\widetilde{\mu_{\varepsilon}}$ coincide con la función $u(0, \varepsilon)$ del teorema de Rudin.

En lo sucesivo vamos a denotar por $G(r)$ a $\mu(B(0, r)), r>0$.

\section{Lema 2.}

Sean $\mu$ una carga de Borel finita definida en $\mathbb{R}^{n}$, y g una función contínuamente diferenciable en ]0, $+\infty$ [, que satisface la condición (3), y tal que

$$
\int_{0}^{+\infty}\left|g^{\prime}(r)\right| d r<+\infty .
$$

Entonces

$$
\widetilde{\mu_{\varepsilon}}=-\int_{0}^{+\infty} \frac{G(r)}{r^{n}}\left(\frac{r}{\varepsilon}\right)^{n+1} g^{\prime}\left(\frac{d r}{r}\right) ; \quad \varepsilon>0 .
$$

\section{Observación}

La condición impuesta sobre la función $g$ se satisface automáticamente si $g(r)$ es una función decreciente, $g(r) \rightarrow 0$ cuando $r \rightarrow+\infty$.

\section{Demostración.}

Podemos asumir sin pérdida de generalidad que $\mu$ es una medida (en el caso general la carga $\mu$ es la diferencia de dos medidas finitas).

Supongamos que $\mu$ es absolutamente continua respecto a la medida de Lebesgue

$$
\mu=f d x, \text { donde } \quad f \in L^{1}\left(\mathbb{R}^{n}\right), \quad f \geq 0 .
$$


Si denotamos por

$$
I(r)=\int_{|\theta|=1} f(r \theta) d \theta
$$

donde $d \theta$ es un elemento de superficie de la bola unidad de $\mathbb{R}^{n}$, obtenemos

$$
\widetilde{\mu_{\varepsilon}}=\frac{1}{\varepsilon^{n}} \int_{\mathbb{R}^{n}} \varphi\left(\frac{x}{\varepsilon}\right) d \mu(x)=\frac{c_{n}}{\varepsilon^{n}} \int_{0}^{+\infty} r^{n-1} I(r) g\left(\frac{r}{\varepsilon}\right) d r .
$$

Ahora, puesto que

$$
G(r)=c_{n} \int_{0}^{r} t^{n-1} I(t) d t
$$

resulta que:

$$
\begin{aligned}
\widetilde{\mu_{\varepsilon}} & =\frac{1}{\varepsilon^{n}} \int_{0}^{+\infty} g\left(\frac{r}{\varepsilon}\right) G^{\prime}(r) d r=\lim _{j \rightarrow+\infty} \frac{1}{\varepsilon^{n}} \int_{0}^{r_{j}} g\left(\frac{r}{\varepsilon}\right) G^{\prime}(r) d r \\
& =\lim _{j \rightarrow+\infty}\left[\frac{1}{\varepsilon^{n}}\left(G\left(r_{j}\right) g\left(\frac{r_{j}}{\varepsilon}\right)-G\left(0^{+}\right) g(0)\right)-\frac{1}{\varepsilon^{n+1}} \int_{0}^{r_{j}} g^{\prime}\left(\frac{r}{\varepsilon}\right) G(r) d r\right] \\
& =\frac{-1}{\varepsilon^{n+1}} \int_{0}^{+\infty} g^{\prime}\left(\frac{r}{\varepsilon}\right) G(r) d r
\end{aligned}
$$

donde la sucesión $\left(r_{j}\right)$ es escogida de tal forma que a la vez se cumplan las condiciones $r_{j} \rightarrow$ $+\infty$ y $g\left(\frac{r_{j}}{\varepsilon}\right) \rightarrow 0$. (Observemos que la existencia de la sucesión $\left(r_{j}\right)$ está garantizada por (3).)

De esta manera terminamos la demostración del lema para el caso en que $\mu$ es una medida absolutamente continua respecto a la medida de Lebesgue.

En el caso general construimos una sucesión $\left(\mu_{j}\right)_{j=1}^{\infty}$ de medidas absolutamente continuas respecto a la medida de Lebesgue, que convergen débilmente a $\mu$. (En calidad de $\mu_{j}$ se puede tomar $\mu * \varphi_{j}$, donde $\varphi_{j}(x)=2^{j n} \varphi\left(2^{j} x\right)$, con $\varphi$ una función de clase $\mathcal{C}^{\infty}$ que satisface $\int \varphi=1$.)

Es inmediato que para cada bola $\mathcal{B}$ cuya frontera tenga $\mu$-medida nula debe cumplirse que

$$
\lim _{j \rightarrow \infty} \mu_{j}(\mathcal{B})=\mu(\mathcal{B}) .
$$

Ahora como $\mu$ es una carga finita, se sigue que el conjunto de los $r$ para los cuales $\mu(\partial B(0, r))>0$ es a lo sumo numerable, y por lo tanto

$$
\lim _{j \rightarrow \infty} G_{j}(r)=G(r)
$$

casi por doquier (con respecto a la medida de Lebesgue), donde

$$
G_{j}=\mu_{j}(B(0, r)) .
$$

Finalmente, en la igualdad

$$
\widetilde{\left(\mu_{j}\right)_{\varepsilon}}=-\int_{0}^{+\infty} \frac{G_{j}(r)}{r^{n}}\left(\frac{r}{\varepsilon}\right)^{n+1} g^{\prime}\left(\frac{d r}{r}\right)
$$


la condición (4): $G_{j}(r) \leq$ var $\mu_{j} \leq C$, permite aplicar el teorema de Lebesgue según el cual se pueden intercambiar límite e integral, y de esta forma la igualdad (5) queda demostrada.

Utilizando la notación del lema anterior podemos demostrar el siguiente teorema "abeliano".

\section{Teorema 3.}

Supongamos que se cumplen las condiciones del Lema 2, y que

$$
\int_{0}^{+\infty} r^{n}\left|g^{\prime}(r)\right| d r<+\infty
$$

Sea

$$
M(r)=\frac{G(r)}{m(B(0, r))}=\frac{G(r)}{r^{n}} .
$$

Si existe $\lim _{r \rightarrow 0} M(r)=L$ y es finito, entonces existe

$$
\lim _{\varepsilon \rightarrow 0} \widetilde{\mu_{\varepsilon}}=L
$$

\section{Demostración.}

Tenemos que

$$
\widetilde{\mu_{\varepsilon}}=-\int_{0}^{+\infty} M(r)\left(\frac{r}{\varepsilon}\right)^{n+1} g^{\prime}\left(\frac{r}{\varepsilon}\right) \frac{d r}{r}=-\int_{0}^{+\infty} M(\varepsilon r) r^{n+1} g^{\prime}(r) \frac{d r}{r} .
$$

La función $M$ es acotada ya que por una parte $\sup G<+\infty$ y por otro lado también existe el límite $\lim _{r \rightarrow 0} M(r)$.

Como por la condición (6)

$$
\int_{0}^{+\infty}\left|g^{\prime}(r)\right| r^{n} d r<+\infty
$$

podemos aplicar el teorema de Lebesgue, i.e.

$$
\begin{aligned}
\lim _{\varepsilon \rightarrow 0} \widetilde{\mu_{\varepsilon}} & \left.=-\int_{0}^{+\infty} L r^{n} g^{\prime}(r) d r=-\left(g(r) r^{n}\right]_{0}^{+\infty}-n \int_{0}^{+\infty} g(r) r^{n-1}\right) \cdot L \\
& =L \int_{0}^{+\infty} n r^{n-1} g(r) d r=L \int_{\mathbb{R}^{n}} \varphi(x) d \mu=L
\end{aligned}
$$

Observemos que (3) implica que $g(r)=o\left(\frac{1}{r^{n}}\right)$ cuando $r$ tiende a $+\infty$, y por consiguiente el término $\left.g(r) r^{n}\right]_{0}^{+\infty}$ se reduce a 0 . 
En lo que sigue queremos ocuparnos del siguiente problema: ¿para cuáles funciones $g$ es cierto el teorema tauberiano?, i.e. ¿para cuáles $g$ se tiene que

$$
\lim _{\varepsilon \rightarrow 0} \widetilde{\mu_{\varepsilon}}=L<+\infty
$$

implica que

$$
\lim _{r \rightarrow 0} M(r)=L
$$

bajo la suposición que $\mu$ es una medida?

\section{Sección 2.}

Sea $\Phi(r)=-r^{n+1} g^{\prime}(r)$.

Denotemos por $\hat{\Phi}$ la transformada de Melin de la función $\Phi$ :

$$
\hat{\Phi} \stackrel{\text { def }}{=} \int_{0}^{+\infty} \Phi(u) u^{-i y} \frac{d u}{u}, y \in \mathbb{R} .
$$

El principal resultado de este trabajo es el siguiente teorema.

\section{Teorema 4.}

Sea $\mu$ una medida de Borel en $\mathbb{R}^{n}, g$ una función que satisface las condiciones (3) y (6), y tal que $g(0) \neq 0$. Si

$$
\hat{\Phi}(y) \neq 0, \forall y \in \mathbb{R}
$$

entonces la condición (7) implica (8).

\section{Observación}

Si suponemos que $g(r)$ es decreciente, $g(r) \rightarrow 0$, cuando $r \rightarrow+\infty$, entonces de la condición (3) se sigue la condición (6), y la condición $g(0) \neq 0$ se verifica automáticamente.

\section{Demostración.}

Es claro que si $\epsilon>0$, entonces podemos escribir

$$
\widetilde{\mu}_{\varepsilon}=(M * \Psi)(\varepsilon),
$$

donde $\Psi(r)=\Phi\left(\frac{1}{r}\right)$, y $*$ denota la convolución en el grupo multiplicativo $\mathbb{R}_{+}^{*}$ de medida invariante $\frac{d r}{r}$, i.e.

$$
M * \Psi=\int_{0}^{+\infty} M\left(\frac{\varepsilon}{r}\right) \Psi(r) \frac{d r}{r} .
$$


Si se cumple la condición (7), entonces la función $M$ es acotada. En efecto, puesto que $g(0) \neq 0$, podemos suponer sin pérdida de generalidad que $g(r)>c>0$, para $0<r<\epsilon$, y en consecuencia

$$
\widetilde{\mu}_{\varepsilon} \geq c \frac{\mu(B(0, \varepsilon))}{\varepsilon^{n}},
$$

de donde $M$ es acotada cerca de 0 . La acotación de $M$ cerca de $+\infty$ es evidente.

La función $\Psi$ es sumable en el grupo $\mathbb{R}_{+}^{*}$ según la medida $\frac{d r}{r}$, ya que de acuerdo a (6)

$$
\begin{aligned}
\int_{0}^{+\infty}|\Psi(r)| \frac{d r}{r} & =\int_{0}^{+\infty}\left|\Phi\left(\frac{1}{r}\right)\right| \frac{d r}{r}= \\
& =\int_{0}^{+\infty}|\Phi(r)| \frac{d r}{r}=\int_{0}^{+\infty}\left|g^{\prime}(r)\right| r^{n} d r<+\infty .
\end{aligned}
$$

Aplicando el Teorema Tauberiano de Wiener, de las condiciones (9) y (7) obtenemos que:

$$
\lim _{\varepsilon \rightarrow 0}(M * f)(\varepsilon)=L, \quad \forall f \in L^{1}\left(\mathbb{R}_{+}^{*}, \frac{d r}{r}\right) .
$$

De la positividad de la carga $\mu$, se tiene que $r^{N} M(r)$ es una función monótona, ya que si $1<r<\gamma$, entonces:

$$
\left(\frac{t}{r}\right)^{n} M\left(\frac{t}{r}\right) \leq t^{n} M(t),
$$

y

$$
M\left(\frac{t}{r}\right) \leq r^{n} M(t) \leq \gamma^{n} M(t)
$$

De la misma manera, si $0<\gamma^{-1}<r<1$, tenemos que:

$$
M\left(\frac{t}{r}\right) \geq r^{n} M(t) \geq \gamma^{-n} M(t) .
$$

Sean ahora $f_{1}$ y $f_{2}$ dos funciones continuas, no negativas, concentradas en $[1, \gamma]$ y $\left[\gamma^{-1}, 1\right]$ respectivamente, y tales que

$$
\int_{0}^{+\infty} \frac{f_{1}(r)}{r} d r=\int_{0}^{+\infty} \frac{f_{2}(r)}{r} d r=1
$$

Entonces, la condición (11) se tiene

$$
\left(M * f_{1}\right)(\varepsilon)=\int_{1}^{\gamma} M\left(\frac{\varepsilon}{r}\right) f_{1}(r) \frac{d r}{r} \leq \gamma^{n} M(\varepsilon),
$$

de donde se sigue que

$$
\gamma^{-n}\left(M * f_{1}\right) \leq M(\varepsilon),
$$

y por $(10)$

$$
\gamma^{-n} \lim _{\varepsilon \rightarrow 0}\left(M * f_{1}\right) \leq \liminf _{\varepsilon \rightarrow 0} M(\varepsilon) .
$$


De esta forma tenemos

$$
\gamma^{-n} L \leq \liminf _{\varepsilon \rightarrow 0} M(\varepsilon), \forall \gamma>1
$$

y concluimos que

$$
L \leq \liminf _{\varepsilon \rightarrow 0} M(\varepsilon)
$$

De la misma manera, utilizando las condiciones (10) y (12), así como la función $f_{2}$ en vez de $f_{1}$, encontramos que

$$
\limsup _{\varepsilon \rightarrow 0} M(\varepsilon) \leq \gamma^{n} L, \forall \gamma>1
$$

de donde

$$
\limsup _{\varepsilon \rightarrow 0} M(\varepsilon) \leq L
$$

y el teorema ha sido demostrado.

\section{Referencias}

[1] Gehring, F. W. (1960) "Harmonic functions and tauberian theorems", London Math. Soc. 10(3): 88-166.

[2] Gehring, F. W. (1957) "The Fatou theorem and its converse", Bull. Amer. Math. Soc. 85: $106-121$.

[3] De Guzmán, M. (1975) "Differentiation of integrals in $\mathbb{R}^{n}$ ", Lecture Notes in Mathematics 481, Springer-Verlag.

[4] Hewitt, E.; Ross, K. A. (1963 y 1970) Abstract Harmonic Analysis, vols. I y II, Springer-Verlag.

[5] Loomis, L. H. (1953) An Introduction to Abstract Harmonic Analysis, Van Nostrand.

[6] Loomis, L. H.; Widdner, D. V. (1942) "The Poisson integral representation of functions which are positive and harmonic in a half plane", Duke Math. Journal 9: 643645.

[7] Rudin, W. (1979) "Tauberian theorems for positive harmonic functions", Bull. Amer. Math. Soc.

[8] Wiener, N. (1932) "Tauberian theorems", Ann. of Math. 33(2): 1-100, 787. 